\title{
OPTIMAL HÖLDER MEAN INEQUALITY FOR THE COMPLETE ELLIPTIC INTEGRALS
}

\begin{abstract}
YUN HUA
Abstract. In this paper, we prove that $H_{p}\left(\mathscr{K}(r), \mathscr{K}\left(r^{\prime}\right)\right) \geqslant \mathscr{K}(\sqrt{2} / 2)$ and $H_{q}\left(\mathscr{K}(r), \mathscr{K}\left(r^{\prime}\right)\right) \leqslant$ $\mathscr{K}(\sqrt{2} / 2)$ for all $r \in(0,1)$ if and only if $p \geqslant 1-4[\mathscr{K}(\sqrt{2} / 2)]^{4} / \pi^{2}=-3.789 \cdots$ and $q \leqslant$ $(\log 2) /[\log (\pi / 2)-\log \mathscr{K}(\sqrt{2} / 2)]=-4.1805 \cdots$, where $H_{p}(x, y)$ denotes the Hölder mean of order $p$ of two positive numbers $x$ and $y, r^{\prime}=\sqrt{1-r^{2}}$, and $\mathscr{K}(r)$ denotes the complete elliptic integral of the first kind, respectively.

Mathematics subject classification (2010): Primary 26E60.

Keywords and phrases: Complete elliptic integrals, Hölder mean, inequality.
\end{abstract}

\section{REFERENCES}

[1] H. Alzer AND S. L. QIU, Monotonicity theorems and inequalities for the complete elliptic integrals, J. Comput. Appl. Math. 172 (2004), 289-312.

[2] G. D. Anderson, S.-L. QiU, M. K. Vamanamurthy, Elliptic integral inequalities, with applications, Constr. Approx. 14, 2 (1998), 195-207.

[3] M. K. Vamanamurthy and M. Vuorinen, Inequalities for means, J. Math. Anal. Appl. 183 (1994), 155-166.

[4] G. D. Anderson, M. K. Vamanamurthy and M. Vuorinen, Functional inequalities for complete elliptic integrals and their ratios, SIAM J. Math. Anal. 21 (1990), 536-549.

[5] G. D. Anderson, M. K. Vamanamurthy and M. Vuorinen, Functional inequalities for hypergeometric functions and complete elliptic integrals, SIAM J. Math. Anal. 23 (1992), 512-524.

[6] G. D. Anderson, S. L. Qiu, M. K. Vamanamurthy and M. Vuorinen, Generalized elliptic integrals and modular equations, Pacific J. Math. 192 (2000), 1-37.

[7] S. ANDRÁS AND Á. BARICZ, Bounds for complete elliptic integral of the first kind, Expo. Math. 28 (2010), 357-364.

[8] G. D. Anderson, M. K. Vamanamurthy and M. Vuorinen, Conformal Invariants, Inequalities, and Quasiconformal Maps, John Wiley \& Sons, New York, 1997.

[9] Miao-Kun Wang, Yu-Ming Chu, Ye-Fang Qiu, Song-Liang Qiu, An optimal power mean inequality for the complete elliptic integrals, Applied Mathematics Letters 24 (2011), 887-890.

[10] Yu-Ming ChU, Ye-FANG QIU AND MiAO-Kun WANG, Hölder mean inequalities for the complete elliptic integrals, Integral Transforms and Special Functions, 23,7 (2012), 521-527.

[11] Miao-Kun Wang, Yu-Ming Chu, Song-Liang Qiu, And Yue-Ping Jiang, Convexity of the complete elliptic integrals of the first kind with respect to Hölder means, J. Math. Anal. Appl. 388, 2 (2011), 1141-1146.

[12] Liming Zhou, Songliang QIu, Fei Wang, Inequalities for the generalized elliptic integrals with respect to Hölder means, J. Math. Anal. Appl. 386, 2 (2012), 641-646.

[13] F. Bowman, Introduction to Elliptic Functions with Applications, Dover Publications, New York, 1961.

[14] P. F. Byrd, M. D. Friedman, Handbook of Elliptic Integrals for Engineers and Scientists, SpringerVerlag, New York, 1971.

[15] P. S. Bullen, Handbook of Means and their Inequalities, Kluwer Academic Publishers Group, Dordrecht, 2003. 